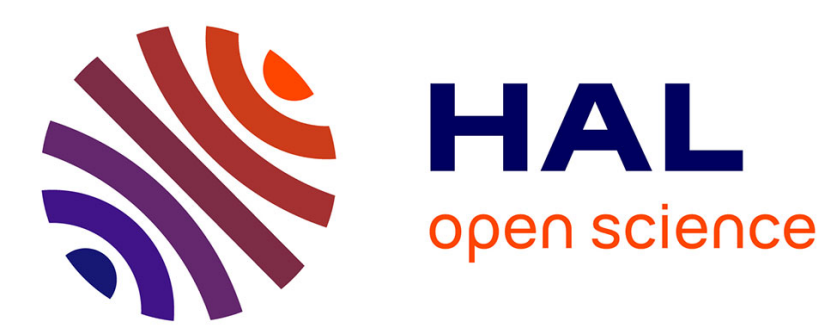

\title{
Tracking Magnetic Rotating Objects by Bipolar Electrochemiluminescence
}

Alice Dauphin, Abdelmounaim Akchach, Silvia Voci, Alexander Kuhn, Guobao Xu, Laurent Bouffier, Neso Sojic

\section{To cite this version:}

Alice Dauphin, Abdelmounaim Akchach, Silvia Voci, Alexander Kuhn, Guobao Xu, et al.. Tracking Magnetic Rotating Objects by Bipolar Electrochemiluminescence. Journal of Physical Chemistry Letters, 2019, 10 (18), pp.5318-5324. 10.1021/acs.jpclett.9b02188 . hal-02509633

\section{HAL Id: hal-02509633 \\ https://hal.science/hal-02509633}

Submitted on 17 Mar 2020

HAL is a multi-disciplinary open access archive for the deposit and dissemination of scientific research documents, whether they are published or not. The documents may come from teaching and research institutions in France or abroad, or from public or private research centers.
L'archive ouverte pluridisciplinaire HAL, est destinée au dépôt et à la diffusion de documents scientifiques de niveau recherche, publiés ou non, émanant des établissements d'enseignement et de recherche français ou étrangers, des laboratoires publics ou privés. 


\title{
Tracking magnetic rotating objects by bipolar electrochemiluminescence
}

\author{
Alice Dauphin, ${ }^{\ddagger}$ Abdelmounaim Akchach, ${ }^{\ddagger}$ Silvia Voci,${ }^{\ddagger}$ Alexander Kuhn,${ }^{\ddagger}$ Guobao Xu, ${ }^{\S}$ \\ Laurent Bouffier, ${ }^{\ddagger}$ Neso Sojic ${ }^{\ddagger} *$ \\ $¥$ University of Bordeaux, Bordeaux INP, ISM, UMR CNRS 5255, 33607 Pessac, France. \\ ${ }^{\S}$ State Key Laboratory of Electroanalytical Chemistry, Changchun Institute of Applied \\ Chemistry, Chinese Academy of Sciences, Changchun, Jilin 130022, P.R. of China. \\ Corresponding Author. E-mail: Neso.Sojic@enscbp.fr
}

\begin{abstract}
There is a very rapid development of original systems that can be remotely controlled or addressed by playing with chemical and physical concepts. Here, we present the synergetic combination of external magnetic and electric fields to promote, in a double contactless mode, the rotational motion and the concomitant generation of light-emission at the level of a goldcoated iron wire. The latter can be moved by rotating magnetic fields. Simultaneously, an electric field induces its remote polarization, which triggers the local generation of electrochemiluminescence (ECL) by bipolar electrochemistry. During rotation, the motion is tracked by changes in ECL intensity as a function of the orientation of the conducting wire in the electric field. The ECL behavior of the rotating bipolar wire is rationalized by considering the angular dependence of the polarization. Unlike previously reported systems, the rotation induces enhanced ECL emission due to the convective flow produced by the motion. This demonstrates that ECL emission can be coupled to magnetically-controlled rotating bipolar objects. Such dual magnetically- and electrically addressable dynamic systems open exciting perspectives for integrating new functions such as imaging and sensing capabilities.
\end{abstract}

\section{TOC Graphics}




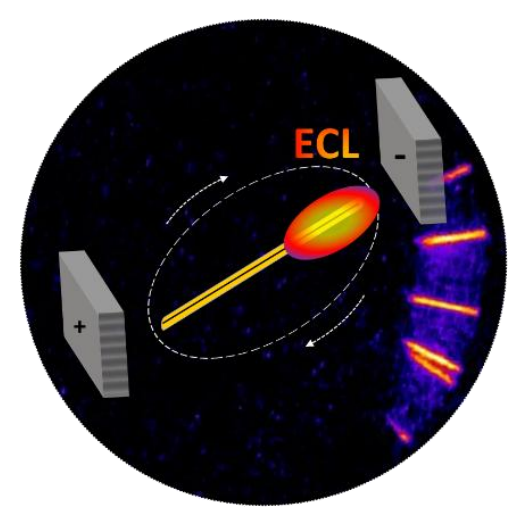

Keywords: Magnetic actuation, wireless electrochemistry, electrochemiluminescence, bipolar electrochemistry, imaging. 
The development of new dynamic systems, for which motion can be remotely controlled, represents an exciting multidisciplinary research area covering various topics, ranging, for example, from analytical chemistry for (bio)sensing applications, to biomedicine with drug delivery, and environmental remediation, etc. ${ }^{1-5}$ These devices can be classified in two main domains. The first one corresponds to fully autonomous systems powered by the catalytic decomposition of solution-borne fuel molecules. The second class, which is the focus of the present study, relies on external sources of energy, especially magnetic fields. Millimeterscale devices manipulated by magnetic fields have been proposed for biological applications. ${ }^{6}$, ${ }^{7}$ Micro- and nano-spinners powered by ultrasound have been investigated and their motion imaged by microvortex advection of nanoparticles. ${ }^{8,9}$ Magnetic systems operating at the micro- of nanometer scale have also been reported. ${ }^{2,10-16}$ However, on the one hand, the main constraint is related to their dimensions, because the magnetic forces driving their motion scales with size. ${ }^{11}$ On the other hand, manipulation concepts based on magnetic fields are contactless, versatile and inexpensive. In this context, this contactless feature provides an essential degree of freedom to such devices and it can be advantageously combined with bipolar electrochemistry (BPE), which is also a wireless methods. Indeed, the synergy of both contactless methods (i.e. steering by the magnetic fields and electrochemical addressing by BPE) offers both, versatility and freedom for the motion of such objects as well as the integration of new features.

BPE is based on the simple application of an electric field across an electrolyte solution. ${ }^{17}$ Thus asymmetric redox activity is promoted at both extremities of a conducting object placed in solution. Indeed, as the conducting object is by definition equipotential, it will experience an inhomogeneous interfacial potential difference with the surrounding solution. This potential difference is proportional to the electric field, to the dimensions of the object and to its orientation in the field. If the electric field is sufficient, redox reactions can be carried out 
at the opposite sides of the conducting objects; oxidation reactions occurring at the anodic pole are compensated by the simultaneous reduction reactions at the cathodic pole. As already mentioned, advantages of BPE are the control provided by the field strength/direction and its versatility, because any kind of conducting object can theoretically be polarized in this wireless mode. This contactless feature of BPE, which constitutes an essential advantage in comparison to classic electrochemistry, has been recently exploited to address either single objects or large ensembles comprising thousands or millions of objects simultaneously with just two feeder electrodes. ${ }^{18-21}$ BPE has been also applied to activate other functions such as light-emitting processes, especially electrochemiluminescence (ECL). ${ }^{22-25}$ ECL is an ultrasensitive and powerful method triggered by an initial electrochemical step. ${ }^{26}$ It is widely used for biosensing and commercialized for immunoassays. Linear motion of an ECL swimmer has been first achieved in a capillary using BPE. ${ }^{18,27}$ ECL emission was intrinsically coupled to the motion. Furthermore, an analytical function was integrated into the swimmer, which was able to detect enzymatically and report by ECL the presence of glucose in a concentration gradient explored during its motion. ${ }^{28}$ Rotational movement of bipolar electrodes has been also imaged by ECL, but the mechanical rotation of the conducting object has been caused by an external motor. ${ }^{29}$ More recently, we reported a wireless bipolar ECL rotor. ${ }^{30}$ The rotor was propelled by the collection of electrochemically-generated $\mathrm{H}_{2}$ bubbles in collector boxes located at the end of the four rotor blades. However, the rotation speed was rather slow since it takes at least $120 \mathrm{~s}$ for one full turn of the rotor. 

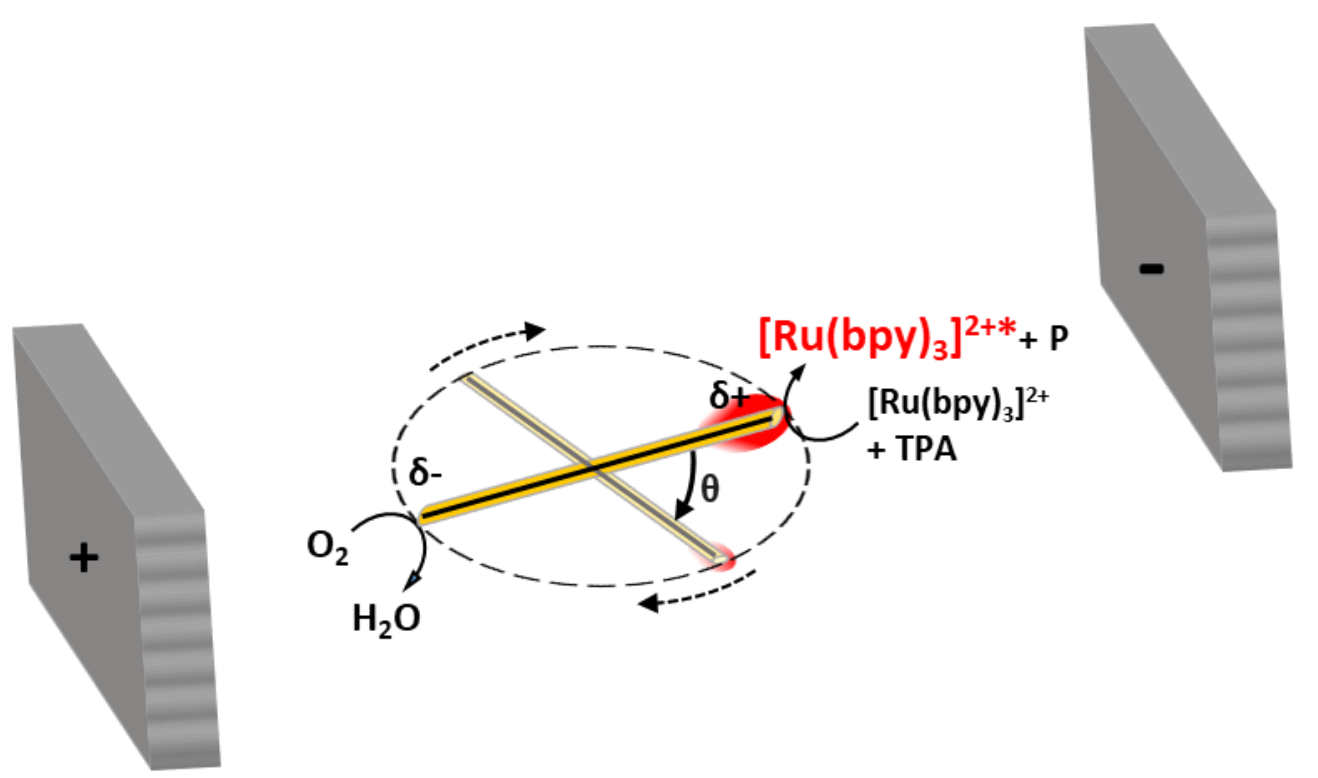

Scheme 1. Principle of the light-emitting magnetically-controlled rotating bipolar electrode. The gold-coated iron wire is placed between two feeder electrodes and acts as a bipolar electrode. Its rotation is induced in the plane perpendicular to the two feeder electrodes, by a magnetic stirrer located under the bipolar electrode. Oxidation of $\left[\mathrm{Ru}(\mathrm{bpy})_{3}\right]^{2+}$ and TPA at the anodic pole $\left(\delta^{+}\right)$generates ECL emission and is coupled to the simultaneous reduction of $\mathrm{O}_{2}$ at the cathodic pole $\left(\delta^{-}\right) . \theta$ is defined as the angle between the longitudinal axis of the goldcoated iron wire when aligned in the electric field generated by the feeder electrodes and a given position during its rotation. P corresponds to a side product of the TPA radicals formed during the ECL process.

Here we present a general strategy which combines both external magnetic and electric fields with ECL emission in a single operating device (Scheme 1). The rotation of the electrode is induced by magnetic forces. The electric field triggers electrochemical reactions at both poles of the bipolar electrode. The resulting ECL emission occurs at the anodic pole, and this optical readout is used to track the motion of the electrode. Conceptually, this object is similar to a classic rotating electrode, but with contactless magnetic control and wireless electrochemical addressing. Finally, enhanced ECL emission is observed due to the convection effects resulting from the rotational motion. The contactless nature of both 
external fields and of the ECL readout maximizes the degrees of freedom of the rotating objects and thus the versatility of the reported approach.

Scheme 1 depicts the principle of the bipolar electrode, which generates ECL emission during its rotation. The light-emitting experiments were performed with a model ECL couple composed of the $\left[\mathrm{Ru}(\mathrm{bpy})_{3}\right]^{2+}$ luminophore and the sacrificial tri-n-propylamine (TPA) coreactant, in a PBS solution ( $\mathrm{pH}$ 7.4). The open bipolar electrochemical set-up consists of a gold-coated iron wire positioned between two planar stainless steel feeder electrodes. We selected iron as the core material for the bipolar electrode because its ferromagnetic properties enable its magnetic manipulation. However, in a classic 3-electrode configuration, we have not been able to generate ECL emission on the iron wire with the $\left[\mathrm{Ru}(\mathrm{bpy})_{3}\right]^{2+} / \mathrm{TPA}$ system. Indeed, the ECL process is highly dependent on the electrode material; glassy carbon or gold are usually employed with this reference ECL system. ${ }^{31}$ Therefore, to keep the ferromagnetic properties required for the magnetic rotation and to confer ECL capabilities, the iron wire was sputter-coated with a thin gold layer. This gold surface allows efficient oxidation of $\left[\mathrm{Ru}(\mathrm{bpy})_{3}\right]^{2+}$ and TPA to generate strong ECL signals. The two feeder electrodes generate an electric field that polarizes the gold-coated iron object in a wireless manner and thus this later acts as a bipolar electrode. When a sufficient electric field is imposed, the oxidation of the ECL reagents occurs at the anodic pole of the wire facing the feeder cathode and it leads to light emission. Oxidation reactions are counter-balanced by the simultaneous reduction of $\mathrm{O}_{2}$ at the cathodic pole of the wire facing the feeder anode. The rotation of the bipolar electrode is induced by a magnetic stirrer located underneath between both feeder electrodes. The rotation speed of the gold-coated iron wire is thus easily tunable. The values of the rotation rate explored in this work ranged from 85 to $255 \mathrm{rpm}$ and are several orders of magnitude higher than the maximum value reached in a previous work with a bipolar rotor (120 s for one 
full turn). ${ }^{30}$ This major improvement is related to the different nature of the energy and mechanisms employed to rotate the bipolar electrode. The angle $\theta$ is defined as the angle between the electric field (i.e. axis perpendicular to both planar feeder electrodes) and a given position during the rotational course of the conducting wire. In other words, the value of $\theta$ is 0 when the longitudinal axis of the iron wire is aligned with the electric field. It is noteworthy that the exact position of the gold-coated wire between the feeder electrodes is not an essential point because the polarization potential only depends on the length and orientation of the bipolar electrode.
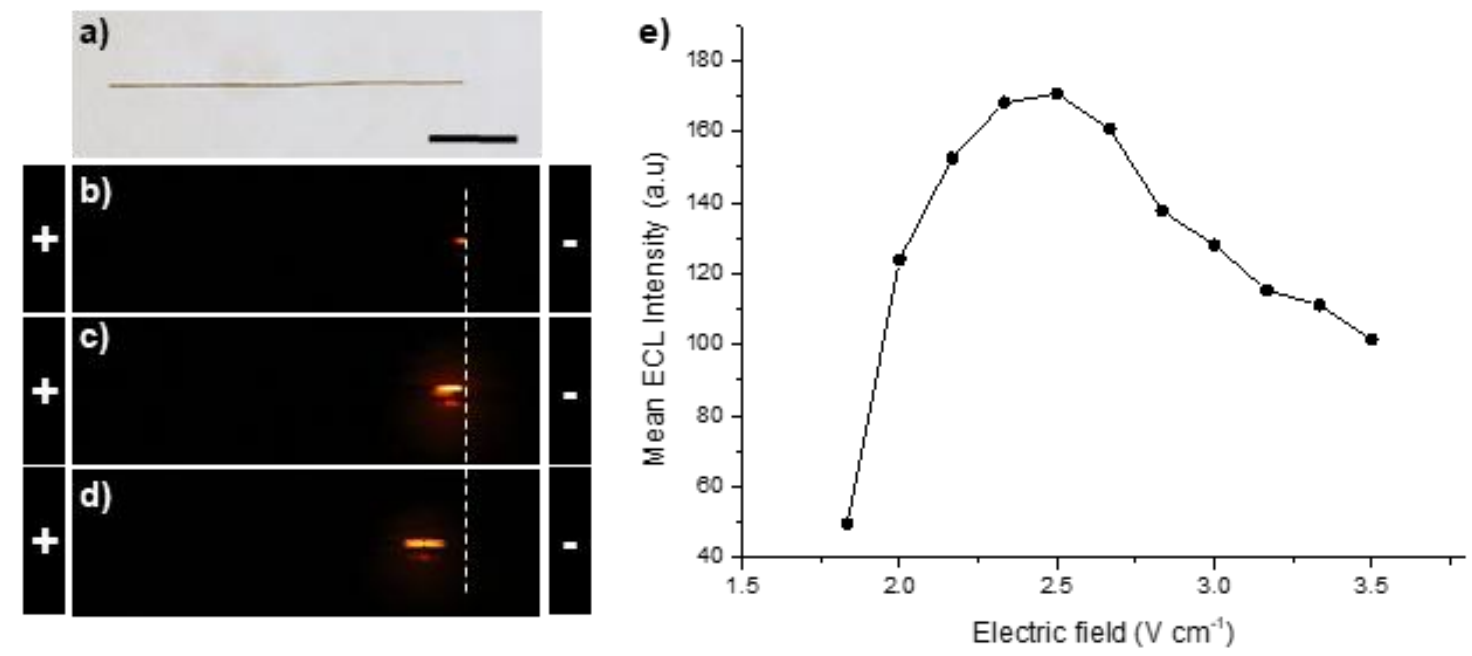

Figure 1. Effect of the electric field on the ECL emission at the magnetic bipolar electrode. Top-view images of the gold-coated iron wire (a) under white light before applying the electric field and (b-d) of the ECL emission in the dark at $\theta=0$ after applying an electric field: of (b) $1.83 \mathrm{~V} \cdot \mathrm{cm}^{-1}$, (c) $2 \mathrm{~V} \cdot \mathrm{cm}^{-1}$, and (d) $2.17 \mathrm{~V} \cdot \mathrm{cm}^{-1}$. Feeder electrodes are symbolically represented. The vertical dashed line materializes the position of the extremity of the anodic pole of the bipolar electrode. (e) Mean ECL intensity as a function of the electric field applied by the feeder electrodes. ECL images were acquired in a PBS (pH 7.4) solution containing $1 \mathrm{mM}\left[\mathrm{Ru}(\mathrm{bpy})_{3}\right]^{2+}$ and $100 \mathrm{mM}$ TPA. The distance between the feeder electrodes was $6 \mathrm{~cm}$ and the bipolar ECL-emitting electrode was placed in the middle. Scale bar: $5 \mathrm{~mm}$. 
A first set of experiments has been performed to study the influence of the electric field strength on the ECL signal (Figure 1). For this, ECL images were recorded in a static mode (i.e. without rotation) with the wire aligned with the electric field $(\theta=0)$. ECL emission starts to appear at the extremity of the wire for a value of $1.83 \mathrm{~V} \mathrm{~cm}^{-1}$ (Figure 1b). With increasing electric fields, the ECL-emitting region expands progressively towards the middle of the bipolar electrode (Figure 1c) because the polarization threshold required for ECL emission is reached also at this position alongside the bipolar electrode. ${ }^{32-34}$ Then, for higher values, the ECL domain moves progressively from the extremity to the middle of the wire (Figure 1d). Such a behavior has been already observed on carbon swimmers and rationalized considering the water oxidation that competes locally with the oxidation of the ECL reagents. ${ }^{18}$ At the anodic extremity of the wire, the oxidation of water provokes a local $\mathrm{pH}$ decrease, which leads to a loss of ECL signal due to the protonation of TPA. ${ }^{35,36}$ When even higher electric fields were applied, the ECL intensity decreases progressively due to the stronger variations of $\mathrm{pH}$ (Figure 1e). To avoid such effects related to $\mathrm{pH}$ variations and also to keep the ECL intensity high at the extremity of the bipolar electrode, we selected a compromise value of $2 \mathrm{~V}$ $\mathrm{cm}^{-1}$ for the further experiments.

Then we investigated the distribution of ECL intensity as a function of the angle during the rotation of the gold-coated iron wire (Figure 2). Rotational motion is tracked by the change in ECL intensity as a function of the orientation of the conducting wire in the electric field (Video S1). For this imaging experiments, a commercial CCD camera recording 50 images per second was employed. The rotation speed was set at $150 \mathrm{rpm}$. It means that each image represents the emitted luminescence over an angular displacement of $18^{\circ}$ for this rotation speed. Video S1 shows an example of ECL signals recorded during the rotation. ECL emission was strong enough that it can be observed with naked eyes. ECL was emitted only on the anodic pole of the wire. Video snapshots were extracted from this file and the 
corresponding single ECL images are presented for different angles on Figure S1. To show an ECL map during the rotational motion, Figure 2a displays an image corresponding to ECL emission recorded during a single half round of the bipolar electrode. In that case, 10 single images were cumulated in a post-recording processing with the ImageJ software to obtain Figure 2a. It means that the intensity of each pixel has been added over 10 successive images to construct Figure 2a. We selected a half round because it corresponds to a single passage of one of the extremity of the bipolar electrode in front of the cathodic feeder electrode. Since both extremities of the gold-coated wire are equivalent, both can generate ECL light when they are close to the horizontal position. It means that 2 ECL "events" occurs for a single round. The spatial extension of the light-emitting region as well as its intensity increase progressively when the wire is gradually aligned with the electric field. Figure 2 a shows that ECL is emitted only on the right side of the image when the extremity of the conducting wire faces the cathodic feeder electrode. 

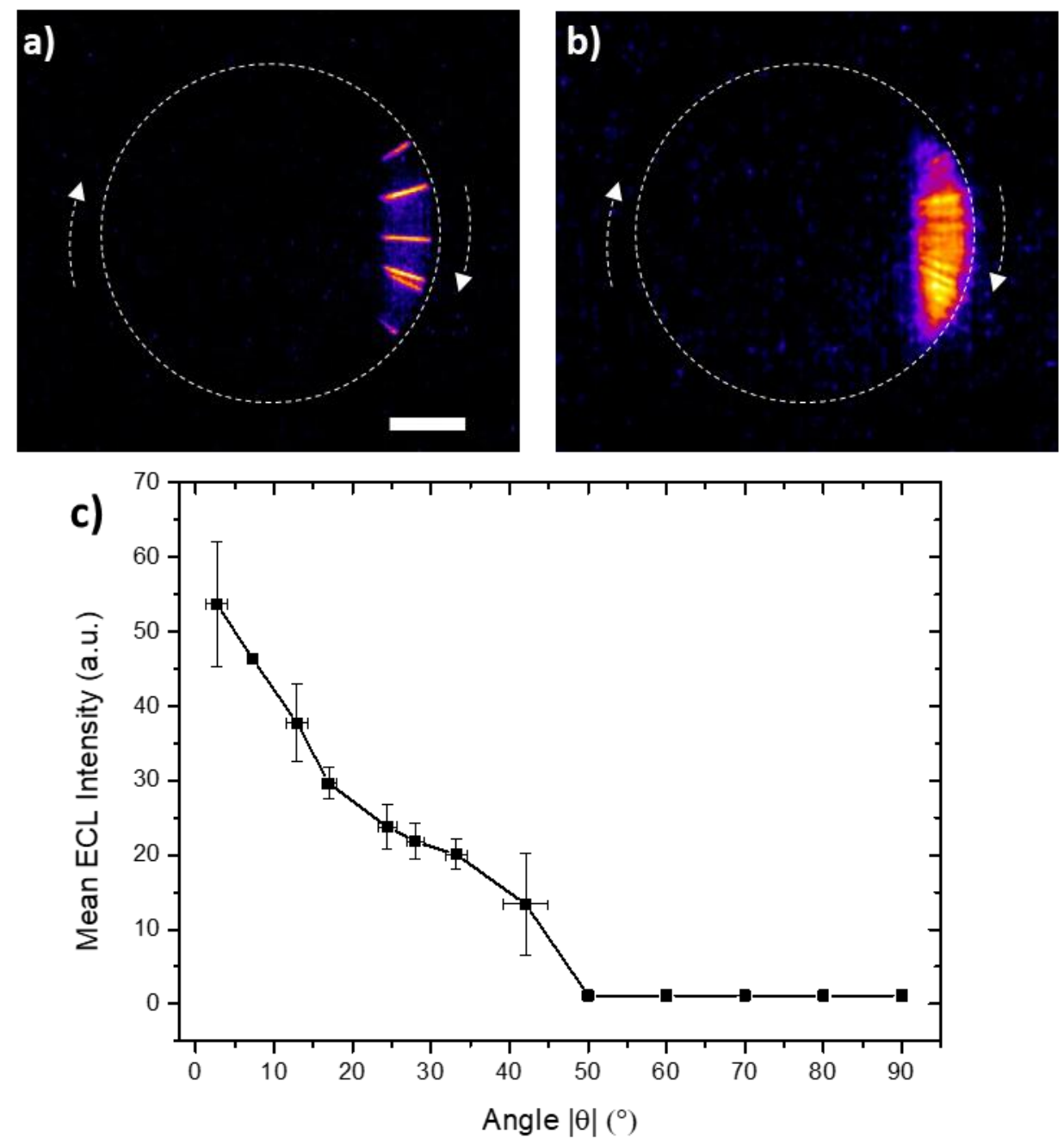

Figure 2. Influence of the angle on the spatial distribution of the ECL emission. (a-b) Cumulated images of the magnetic bipolar electrode showing the ECL distribution during the rotation. ECL images were accumulated during (a) a single half-round or (b) 32.5 rounds corresponding to $13 \mathrm{~s}$. The dashed line materializes the trajectory of the rotating electrode. The anodic and cathodic feeder electrodes are located on the left and right side of the images, respectively. c) Mean ECL intensity as a function of the absolute values of the different angles (i.e. $|\theta|$ ) during the rotation. ECL images were acquired in a PBS ( $\mathrm{pH} 7.4$ ) solution containing $2 \mathrm{mM}\left[\mathrm{Ru}(\mathrm{bpy})_{3}\right]^{2+}$ and $5 \mathrm{mM}$ TPA at a rotation speed of $150 \mathrm{rpm}$ with an applied electric field of $2 \mathrm{~V} \cdot \mathrm{cm}^{-1}$. The images were coded with false colors. Scale bar: $5 \mathrm{~mm}$.

To obtain an average map of the global ECL distribution, we cumulated the images for $13 \mathrm{~s}$, corresponding to 32.5 rounds or 650 images (Figure 2b). It shows clearly the angular dependence of the ECL light, which is averaged over 65 ECL "events". From the ECL images, we can extract and plot the variations of the ECL intensity as a function of the angle $\theta$ 
(Figure 2c). ECL intensity is maximal at $\theta=0$ and then decreases monotonically until $|\theta| \geq$ $50^{\circ}$ where ECL light was not detected anymore. This ECL map reflects the effects of the orientation of the conducting wire in the electric field $(E)$ and the resulting dependence of the polarization potential difference $\left(\Delta V_{\text {wire }}\right)$ on the angle $\theta$. The value of $\Delta V_{\text {wire }}$ governs the ECL behavior of the bipolar electrode during its rotation. $\Delta V_{\text {wire }}$ is directly related to the electric field strength, the length of the conducting wire $L$ and the angle $\theta$, as described by the simple relation: ${ }^{17,37-39}$

$$
\Delta V_{\text {wire }}=E \times L \times \cos \theta
$$

Depending on the orientation of the wire in the electric field, this polarization potential difference varies. Indeed, the magnitude of the electric field experienced by the wire is given by the $i R$-drop in solution, which depends on its orientation in the applied electric field. From the point of view of BPE, the effective length of the bipolar electrode is the projected length of the object on the axis parallel to the electric field and passing through the rotational axis of the wire. The maximum value of $\Delta V_{\text {wire }}$ is reached when the wire is aligned with the electric field $(\theta=0)$. It explains the maximum ECL intensity observed in this situation. When the wire moves away from this value, polarization decreases progressively and thus the ECL signal follows this pattern. There is no ECL emission when the wire is oriented with an angle $|\theta| \geq$ $50^{\circ}$ (Figure 2c) because then the polarization difference is below the threshold value required to induce both the reduction of $\mathrm{O}_{2}$ and the oxidation of ECL reagents at the opposite extremities of the conducting wire. The ECL behavior of the rotating bipolar wire is thus rationalized by considering the angular dependence of the polarization difference.

Video S1 displays the variations of the ECL intensity during the rotation of the bipolar wire. ECL is turned on and off in a regular manner when the longitudinal axis of the wire approached the position where it is aligned with the electric field. To show the reproducibility of the variations of the luminescence signal, we calculated the mean value of the light 
intensity for each video snapshot. Figure 3 illustrates the variation of the mean ECL intensity as a function of time during the rotational motion for two different speeds. ECL is emitted only when the electric field is switched on at $t=2 \mathrm{~s}$. A series of ECL peaks appears in a very regular manner (Figure 3, top). Each peak corresponds to the passing of the extremity of the bipolar wire in front of the cathodic feeder electrode. As already mentioned, it means that two peaks correspond to a full round of the object. One can see clearly that the height of the peaks are constant and it reflects the excellent stability and reproducibility of the ECL signals in this approach.
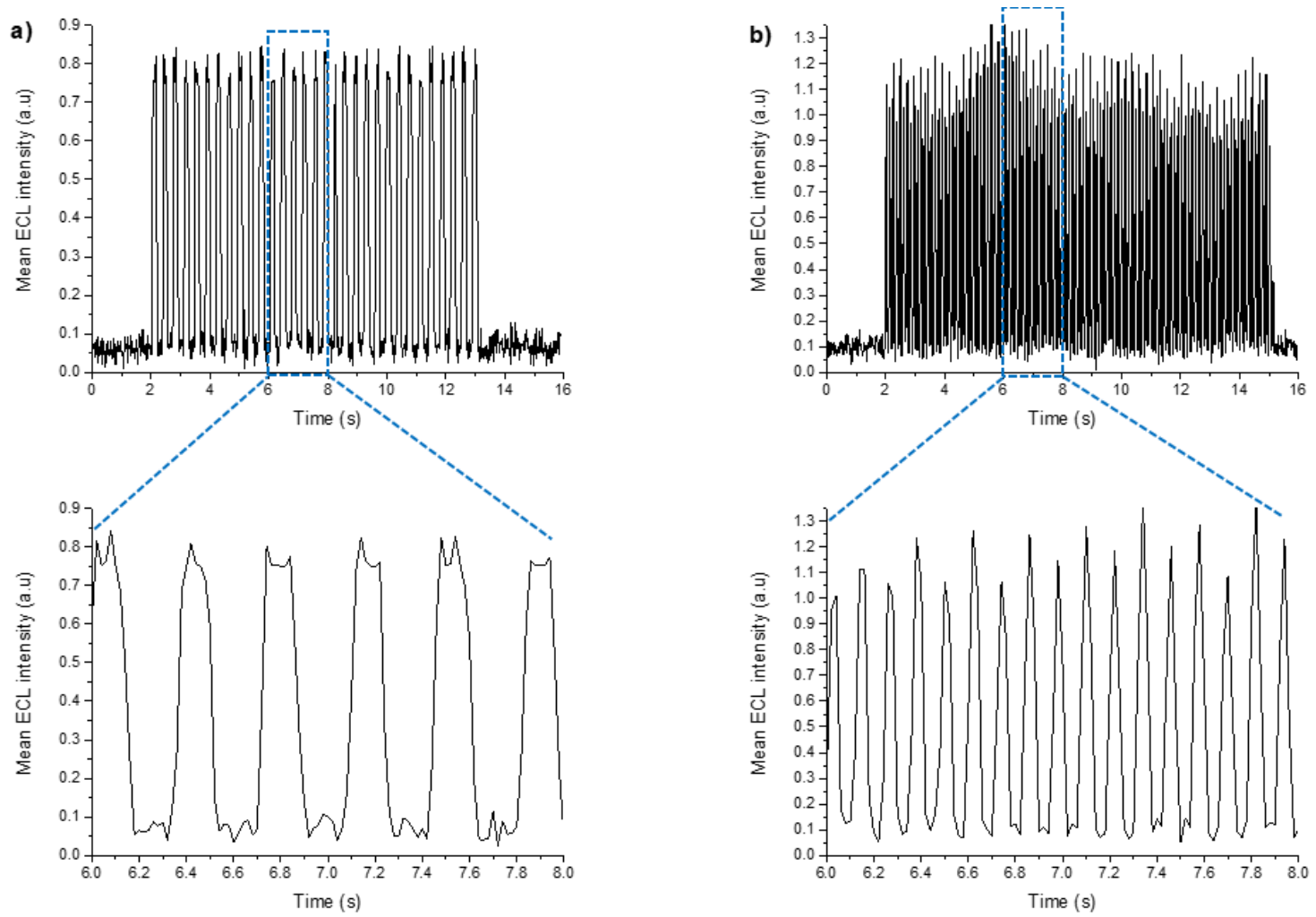

Figure 3. Variations of the mean ECL intensity as a function of time recorded at (a) $85 \mathrm{rpm}$ and (b) $255 \mathrm{rpm}$. The electric field is applied at $\mathrm{t}=2 \mathrm{~s}$. ECL images that were used to measure the mean ECL intensity were acquired in a PBS (pH 7.4) solution containing $2 \mathrm{mM}$ $\left[\mathrm{Ru}(\mathrm{bpy})_{3}\right]^{2+}$ and $5 \mathrm{mM}$ TPA with an applied electric field of $2 \mathrm{~V} \cdot \mathrm{cm}^{-1}$. 
Figure 3a (bottom) allows visualizing better the shape of the peak: ECL intensity raised very rapidly and reached a plateau. The raising time is $\sim 60 \mathrm{~ms}$ at $85 \mathrm{rpm}$. Finally, it decreased as quickly as it has increased due to the constant rotation rate. However, it can be limiting to resolve the peak shape at high rotation speed. Indeed, it is important to keep in mind that the shape is convoluted by the temporal performances of the photodetector. In the present work, we used a commercial CCD camera recording 50 images per second in the video mode. But, this issue can be solved easily by using a high speed CCD camera. The variations of the fullwidth at half-maximum (FWHM) with the rotation speed is discussed in the Supporting Information (Figure S3). By increasing progressively the rotation speed, the ECL intensity as well as the frequency of the peaks increases (Video S2 and Figure S2). For instance, more than 50 ECL peaks are temporally resolved in $10 \mathrm{~s}$ at $255 \mathrm{rpm}$ (Figure $3 \mathrm{~b}$, top). The peak frequency is in very good agreement with the rotation speed displayed by the magnetic stirrer. This figure reveals a uniform statistic distribution of the ECL signals. In addition, the collection of such a high number of ECL peaks may allow improving the signal-to-noise ratio by averaging them and thus the sensitivity of the detection. 


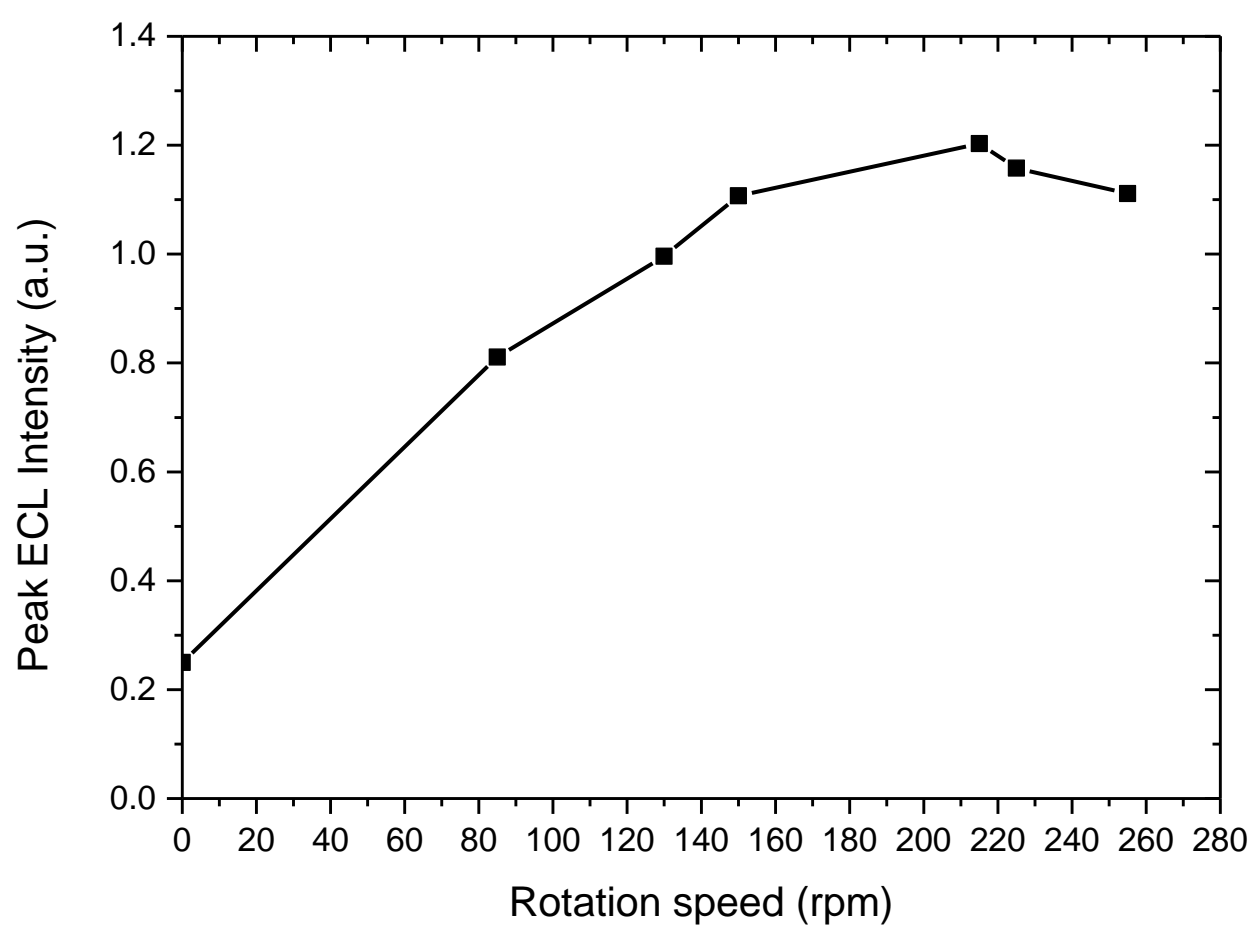

Figure 4. Variations of the peak ECL intensity as a function of the rotation speed. Values were extracted from ECL images acquired in a PBS ( $\mathrm{pH} 7.4)$ solution containing $2 \mathrm{mM}$ $\left[\mathrm{Ru}(\mathrm{bpy})_{3}\right]^{2+}$ and $5 \mathrm{mM}$ TPA in static mode (first point at $0 \mathrm{rpm}$ ) and at a different rotation rates with an applied electric field of $2 \mathrm{~V} \cdot \mathrm{cm}^{-1}$.

The rotational motion of the gold-coated iron wire induced naturally a convection. The presented strategy is conceptually equivalent to a rotating electrode where the convection flows are controlled. ${ }^{40}$ The effects of natural and forced convections are well-studied in electrochemistry because the mass transfer is an essential process associated with the charge transfer. Electrochemical methods involving well-controlled convection of reagents are often called hydrodynamic methods. Steady-state conditions are usually reached very quickly and high precision of the measurements is easily achieved. With the approach reported here based on rotating bipolar wires, the convective mass transport is easily tuned in a contactless manner using the magnetic forces. In a static mode (i.e. without stirring) at $\theta=0$ where polarization and thus the ECL response are maximal, the steady-state ECL intensity is 0.25 . This value is 
compared with peak ECL intensity at different rotation rates on Figure 4 . At $85 \mathrm{rpm}$, the value is 0.81 so the ECL intensity is enhanced more than 3-fold. ECL signals continue to increase with the rotation rate. At higher values than $215 \mathrm{rpm}$, the peak ECL intensity starts to slightly decrease. It is most probably related to the dynamics of the CCD camera, which cannot catch the ECL images sufficiently fast. Indeed, at such high rates, an image averages the ECL signal over $30^{\circ}$, value where ECL starts to decrease already. But, for instance, at $215 \mathrm{rpm}$, the ECL signal is $\sim 5$-fold stronger than in the static mode. ECL is limited by the transport of the reactants, $\mathrm{O}_{2}$ and $\left[\mathrm{Ru}(\mathrm{bpy})_{3}\right]^{2+} / \mathrm{TPA}$, to the cathodic and anodic poles of the bipolar electrode, respectively. By increasing the rotation speed, the convective flow brings more reactants to both extremities of the bipolar electrode, where they are oxidized and reduced. In this way, ECL intensity can therefore be enhanced in a very well-controlled way by changing the motion of the object. It implies also that the angular velocity of the rotating object can be estimated from the ECL intensity using the curve on Figure 4 as a calibration plot.

In conclusion, an original double-remote system has been designed by combining simultaneously the actions of external magnetic and electric fields. They induce the rotational motion of a gold-coated iron wire and its bipolar polarization, respectively. This latter triggers local ECL emission, which is used as an imaging readout method. The rotational course is tracked by the light emission occurring at the anodic pole, preferentially when the wire is aligned with the electric field vector. The ECL distribution reflects the angular dependence of the electrochemical polarization of the object. Finally, ECL emission can be enhanced by the convection resulting from the rotational motion. The versatility of the reported strategy based on magnetic stirring, BPE and ECL could allow the same principles to be applied to other types of conducting objects and at smaller scales. The presented approach could also be 
extended to design a variety of multifunctional systems coupled, for example, to dynamic enzymatic detection or immunoassays.

\section{Experimental section}

Materials and Chemicals: All solutions were prepared with ultrapure water from a Milli-Q dispenser (Millipore, resistivity $18.2 \mathrm{M} \Omega \mathrm{cm}$ at $25^{\circ} \mathrm{C}$ ). All chemicals were purchased from Sigma-Aldrich and used without further purification. ECL solutions were composed of tris(2,2'-bipyridyl)-dichloro ruthenium(II) hexahydrate (powder) and tri-n-propylamine ( $\geq 98 \%$ ) in $100 \mathrm{mM}$ phosphate buffer $\mathrm{pH} 7.4$ (Potassium phosphate mono- and dibasic, ACS reagents, 98+). Stainless steel plates were used as feeder electrodes (Goodfellow, $300 \mathrm{~mm} \times$ $300 \mathrm{~mm} \times 0.25 \mathrm{~mm}$ ) and an iron wire (Goodfellow) coated with a gold layer was used as bipolar electrode. This gold layer was deposited by sputtering (K550X from Quorum Technologies, $35 \mathrm{~mA}, 1 \times 10^{-1} \mathrm{mbar}, 90 \mathrm{~s}$ ). The iron wire (diameter: $150 \mu \mathrm{m}$, length: $20 \mathrm{~mm}$ ) was positioned perpendicular to the gold target and the metallization was performed twice in order to coat both sides of the wire with a gold layer. A power supply (EA Elektro-Automatik, EA-PS 5080-10A, Radiospares) was used to apply a continuous voltage between both feeder electrodes. The rotation was induced by a magnetic stirrer (VWR) and the data were monitored by using a CCD camera (CANON EOS 70D, Objective Canon Macro Lens 100 mm 1:2.8). Images and videos (AVI encoding) were processed with Image $\mathbf{J}$ software. The videos were finally converted to WMV format.

\section{ASSOCIATED CONTENT}


Supporting information. Two multimedia files: Video S1. ECL emission during the rotation at $150 \mathrm{rpm}$ with an applied electric field of $2 \mathrm{~V} \mathrm{~cm}^{-1}$. Video $\mathbf{S 2}$. ECL emission at increasing rotation rates with an applied electric field of $2 \mathrm{~V} \mathrm{~cm}^{-1}$. ECL images recorded at different rotation angles. Variations of the mean ECL intensity as a function of time recorded at different rotation rates. Discussion of the variations of the FWHM with the rotation rate.

\section{AUTHOR INFORMATION}

\section{Corresponding Authors}

*E-mail: Neso.Sojic@enscbp.fr

\section{Notes}

The authors declare no competing financial interest.

\section{Acknowledgements}

We thank the Agence Nationale de la Recherche (Neocastip ANR-15-CE09-0015-03) and CAS President's International Fellowship Initiative (PIFI) for their financial supports. This project has also been funded by the European Research Council (ERC) under the European Union's Horizon 2020 research and innovation program (grant agreement $n^{\circ} 741251$, ERC Advanced grant ELECTRA). The authors wish also to acknowledge the support from the Sino-French international research network "New nanostructured materials and biomaterials for renewable electrical energy sources" for providing facilities. 


\section{References}

1. Mei, Y.; Solovev, A. A.; Sanchez, S.; Schmidt, O. G. Rolled-up Nanotech on Polymers: From Basic Perception to Self-propelled Catalytic Microengines. Chem. Soc. Rev. 2011, 40, 2109-2119.

2. Patiño, T.; Arqué, X.; Mestre, R.; Palacios, L.; Sánchez, S. Fundamental Aspects of EnzymePowered Micro- and Nanoswimmers. Acc. Chem. Res. 2018, 51, 2662-2671.

3. Vilela, D.; Cossío, U.; Parmar, J.; Martínez-Villacorta, A. M.; Gómez-Vallejo, V.; Llop, J.; Sánchez, S. Medical Imaging for the Tracking of Micromotors. ACS Nano 2018, 12, 1220-1227.

4. $\quad$ Palagi, S.; Fischer, P. Bioinspired Microrobots. Nat. Rev. Mater. 2018, 3, 113-124.

5. Wang, W.; Chiang, T.-Y.; Velegol, D.; Mallouk, T. E. Understanding the Efficiency of Autonomous Nano- and Microscale Motors. J. Am. Chem. Soc. 2013, 135, 10557-10565.

6. Martel, S.; Mathieu, J.-B.; Felfoul, O.; Chanu, A.; Aboussouan, E.; Tamaz, S.; Pouponneau, P.; Yahia, L. H.; Beaudoin, G.; Soulez, G.; Mankiewicz, M. Automatic Navigation of an Untethered Device in the Artery of a Living Animal Using a Conventional Clinical Magnetic Resonance Imaging System. Appl. Phys. Lett. 2007, 90, 114105.

7. Kummer, M. P.; Abbott, J. J.; Kratochvil, B. E.; Borer, R.; Sengul, A.; Nelson, B. J. OctoMag: An Electromagnetic System for 5-DOF Wireless Micromanipulation. IEEE Trans. Rob. 2010, 26, 10061017.

8. Balk, A. L.; Mair, L. O.; Mathai, P. P.; Patrone, P. N.; Wang, W.; Ahmed, S.; Mallouk, T. E.; Liddle, J. A.; Stavis, S. M. Kilohertz Rotation of Nanorods Propelled by Ultrasound, Traced by Microvortex Advection of Nanoparticles. ACS Nano 2014, 8, 8300-8309.

9. Sabrina, S.; Tasinkevych, M.; Ahmed, S.; Brooks, A. M.; Olvera de la Cruz, M.; Mallouk, T. E.; Bishop, K. J. M. Shape-Directed Microspinners Powered by Ultrasound. ACS Nano 2018, 12, 29392947.

10. Palagi, S.; Mazzolai, B.; Innocenti, C.; Sangregorio, C.; Beccai, L. How Does Buoyancy of Hydrogel Microrobots Affect Their Magnetic Propulsion in Liquids? Appl. Phys. Lett. 2013, 102, 124102.

11. Han, K.; Shields IV, C. W.; Velev, O. D. Engineering of Self-Propelling Microbots and Microdevices Powered by Magnetic and Electric Fields. Adv. Funct. Mat. 2018, 28, 1705953.

12. Peyer, K. E.; Tottori, S.; Qiu, F.; Zhang, L.; Nelson, B. J. Magnetic Helical Micromachines. Chem. Eur. J. 2013, 19, 28-38.

13. Ghosh, A.; Fischer, P. Controlled Propulsion of Artificial Magnetic Nanostructured Propellers. Nano Letters 2009, 9, 2243-2245.

14. Satterwhite-Warden, J. E.; Kondepudi, D. K.; Dixon, J. A.; Rusling, J. F. Thermal- and Magnetic-Sensitive Particle Flocking Motion at the Air-Water Interface. J. Phys. Chem. B 2019, 123, 3832-3840.

15. Ng, W. M.; Che, H. X.; Guo, C.; Liu, C.; Low, S. C.; Chieh Chan, D. J.; Mohamud, R.; Lim, J. Artificial Magnetotaxis of Microbot: Magnetophoresis versus Self-Swimming. Langmuir 2018, 34, 7971-7980.

16. Katuri, J.; Ma, X.; Stanton, M. M.; Sánchez, S. Designing Micro- and Nanoswimmers for Specific Applications. Acc. Chem. Res. 2017, 50, 2-11.

17. Fosdick, S. E.; Knust, K. N.; Scida, K.; Crooks, R. M. Bipolar Electrochemistry. Angew. Chem. Int. Ed. 2013, 52, 10438-10456.

18. Sentic, M.; Loget, G.; Manojlovic, D.; Kuhn, A.; Sojic, N. Light-Emitting Electrochemical Swimmers. Angew. Chem. Int. Ed. 2012, 51, 11284-11288.

19. Juskova, P.; Neuzil, P.; Manz, A.; Foret, F. Detection of Electrochemiluminescence From Floating Metal Platelets in Suspension. Lab on a Chip 2013, 13, 781-784.

20. Sentic, M.; Arbault, S.; Bouffier, L.; Manojlovic, D.; Kuhn, A.; Sojic, N. 3D Electrogenerated Chemiluminescence: From Surface-Confined Reactions to Bulk Emission. Chem. Sci. 2015, 6, 44334437. 
21. de Poulpiquet, A.; Diez-Buitrago, B.; Dumont Milutinovic, M.; Sentic, M.; Arbault, S.; Bouffier, L.; Kuhn, A.; Sojic, N. Dual Enzymatic Detection by Bulk Electrogenerated Chemiluminescence. Anal. Chem. 2016, 88, 6585-6592.

22. Arora, A.; Eijkel, J. C. T.; Morf, W. E.; Manz, A. A Wireless Electrochemiluminescence Detector Applied to Direct and Indirect Detection for Electrophoresis on a Microfabricated Glass Device. Anal. Chem. 2001, 73, 3282-3288.

23. Gao, W.; Muzyka, K.; Ma, X.; Lou, B.; Xu, G. A Single-Electrode Electrochemical System for Multiplex Electrochemiluminescence Analysis Based on a Resistance Induced Potential Difference. Chem. Sci. 2018, 9, 3911-3916.

24. Bouffier, L.; Ravaine, V.; Sojic, N.; Kuhn, A. Electric Fields for Generating Unconventional Motion of Small Objects. Curr. Opin. Colloid Interface Sci. 2016, 21, 57-64.

25. Bouffier, L.; Manojlovic, D.; Kuhn, A.; Sojic, N. Advances in Bipolar

Electrochemiluminescence for the Detection of Biorelevant Molecular Targets. Curr. Opin. Electrochem. 2019, 16, 28-34.

26. Liu, Z.; Qi, W.; Xu, G. Recent Advances in Electrochemiluminescence. Chem. Soc. Rev. 2015, 44, 3117-3142.

27. Bouffier, L.; Zigah, D.; Adam, C.; Sentic, M.; Fattah, Z.; Manojlovic, D.; Kuhn, A.; Sojic, N. Lighting Up Redox Propulsion with Luminol Electrogenerated Chemiluminescence. ChemElectroChem 2014, 1, 95-98.

28. Sentic, M.; Arbault, S.; Goudeau, B.; Manojlovic, D.; Kuhn, A.; Bouffier, L.; Sojic, N. Electrochemiluminescent Swimmers for Dynamic Enzymatic Sensing. Chem. Commun. 2014, 50, 10202-10205.

29. Eßmann, V.; Clausmeyer, J.; Schuhmann, W. Alternating Current-Bipolar Electrochemistry. Electrochem. Commun. 2017, 75, 82-85.

30. Eßmann, V.; Voci, S.; Loget, G.; Sojic, N.; Schuhmann, W.; Kuhn, A. Wireless Light-Emitting Electrochemical Rotors. J. Phys. Chem. Lett. 2017, 8, 4930-4934.

31. Valenti, G.; Fiorani, A.; Li, H.; Sojic, N.; Paolucci, F. Essential Role of Electrode Materials in Electrochemiluminescence Applications. ChemElectroChem 2016, 3, 1990-1997.

32. Doeven, E. H.; Zammit, E. M.; Barbante, G. J.; Francis, P. S.; Barnett, N. W.; Hogan, C. F. A potential-controlled switch on/off mechanism for selective excitation in mixed electrochemiluminescent systems. Chem. Sci. 2013, 4, 977-982.

33. Doeven, E. H.; Barbante, G. J.; Kerr, E.; Hogan, C. F.; Endler, J. A.; Francis, P. S. Red-GreenBlue Electrogenerated Chemiluminescence Utilizing a Digital Camera as Detector. Anal. Chem. 2014, 86, 2727-2732.

34. Li, H.; Bouffier, L.; Arbault, S.; Kuhn, A.; Hogan, C. F.; Sojic, N. Spatially-resolved multicolor bipolar electrochemiluminescence. Electrochem. Commun. 2017, 77, 10-13.

35. Leland, J. K.; Powell, M. J. Electrogenerated Chemiluminescence : An Oxidative-Reduction Type ECL Reaction Sequence Using Tripropyl amine. J. Electrochem. Soc. 1990, 137, 3127-3131.

36. Badocco, D.; Zanon, F.; Pastore, P. Use of Ru(bpy)32+/Tertiary Aliphatic Amine System Fast Potential Pulses Electrochemiluminescence at Ultramicroelectrodes Coupled to Electrochemical Data for Evaluating E॰ of Amine Redox Couples. Electrochim. Acta 2006, 51, 6442-6450.

37. Mavré, F.; Chow, K.-F.; Sheridan, E.; Chang, B.-Y.; Crooks, J. A.; Crooks, R. M. A Theoretical and Experimental Framework for Understanding Electrogenerated Chemiluminescence (ECL)

Emission at Bipolar Electrodes. Anal. Chem. 2009, 81, 6218-6225.

38. Loget, G.; Zigah, D.; Bouffier, L.; Sojic, N.; Kuhn, A. Bipolar Electrochemistry: From Materials Science to Motion and Beyond. Acc. Chem. Res. 2013, 46, 2513-2523.

39. L.Bouffier, D. Z., N. Sojic, A. Kuhn, Recent advances in bipolar electrochemistry. In Electroanalytical Chemistry: A Series of Advances. Vol. 27, A. J. Bard, C. G. Z., Ed. CRC Press: 2017. 40. Bard, A. J.; Faulkner, L. R., Electrochemical methods. Wiley: New York, 2001. 